\title{
ANALYSIS OF PATTERNS IN TREMOR DIAGNOSIS SPIRAL DRAWINGS FOR AUTOMATED CLASSIFICATION
}

\author{
Andreas Wille ${ }^{1}$, Mohamed Sangaré ${ }^{1}$ and Susanne Winter ${ }^{1}$ \\ ${ }^{1}$ Institut für Neuroinformatik, Ruhr-Universität Bochum, Germany \\ andreas.wille@ini.rub.de
}

\begin{abstract}
Tremor analysis is a common task in diagnosis of movement disorders. In this paper, we present a new method for an automated classification of tremor strength based on digitized spiral drawings. We used statistical measurements on relative line orientations, which are calculated on the full image. Therefore, this approach is insensitive to drawing errors (like line crossings or missing line parts). On a test set of 109 clinical spiral drawings the difference within the calculated values for different levels of tremor strength was highly significant and allowed a fast and straightforward classification.
\end{abstract}

Keywords: Tremor, spiral drawing, classification, orientation histogram

\section{Introduction}

Tremor is an unintended, rhythmic, oscillatory muscle movement in one or more parts of the body. A light tremor is physiologically normal for humans. This tremor can become visible under certain conditions, e.g., stress, anxiety or excitement. Other reasons for tremor are diseases like the Parkinson disease or affections of the cerebellum. As tremor is the most common movement disorder a differentiated classification of tremor and tremor strength is necessary. A common practice is the individual classification of patient movements in relation to some standard by an experienced neurologist. Another method is the comparison between spiral drawings and standardized spiral images depicting effects of ten different levels of tremor [1]. As the intra- and interindividual variation in this classification is high, an automated evaluation of the drawings could help to perform the evaluation more objectively and allow a precise monitoring of tremor progress.

Kraus and Hoffmann [2] developed a method to automatically analyze the tremor amplitude in spiral drawings. However, in this method problems occur whenever it is not possible to fit an ideal spiral-curve to the drawing due to crossings or gaps in the drawn line.

To overcome these drawbacks we already examined some measures of two-dimensional histograms [3] and found them to be quite helpful and suitable for classification of tremor strength. Yet, this method still requires visual inspection of the two-dimensional histograms, which proved to be difficult without some experience.

As a logical next step, we present a method to quantify the differences between tremor strengths in spiral drawings by computing a value which correlates to the variation of the

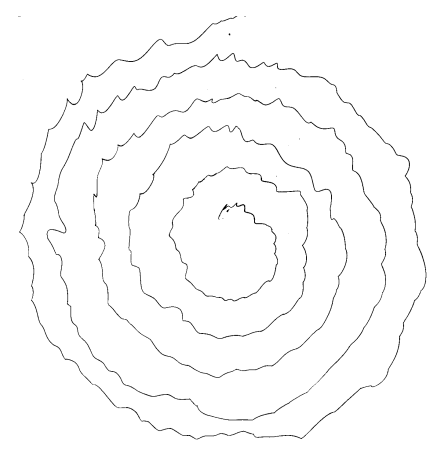

Figure 1: Spiral image after preprocessing

relative orientation of the drawn line with respect to the spiral center.

\section{Methods}

For image creation we follow the standard procedure according to Bain and Findley [1], i.e. the patient draws a spiral on a piece of paper by following an ideal image of Archimedes' Spiral (which is by some means projected on the paper) as close as possible. Afterwards the resulting image is digitized and the drawing area is extracted. To avoid miscounts the image must not contain any additional information except the spiral drawing. An example for such an image is shown in Fig. 1. By application of a Sobel filter for both horizontal and vertical direction the gradient and it's orientation $\phi$ are computed for every foreground image pixel $I_{f}$ (see Eq. 1).

$$
\phi(x, y)=\arctan \left(\frac{\nabla_{y} I_{f}(x, y)}{\nabla_{x} I_{f}(x, y)}\right)
$$

In this case Sobel filters of size $7 \times 7$ were used to achieve an appropriate level of detail in the orientation. Additionally, the angle $\alpha$ between each pixel and the image center (which is equivalent to the center of the spiral) relative to a horizontal line through the center is calculated. A subtraction of these angles according to Eq. 2 results in a measure for the relative orientation $\theta$ of all lines.

$$
\theta=\phi-\alpha
$$

After the relative orientation is computed for all foreground pixels, mean and standard deviation are calculated. Alternatively the acquired set is visualized in form of a histogram using one bin for every ten degrees. 


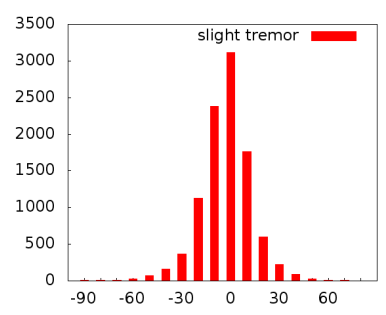

(a) slight

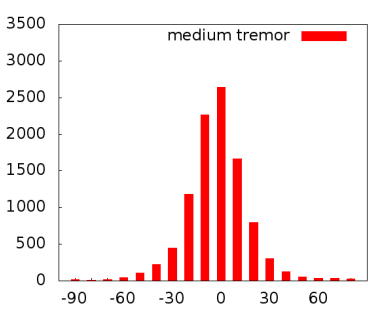

(b) medium

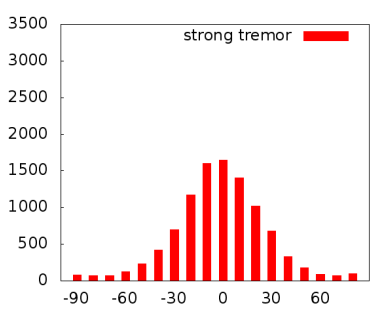

(c) strong

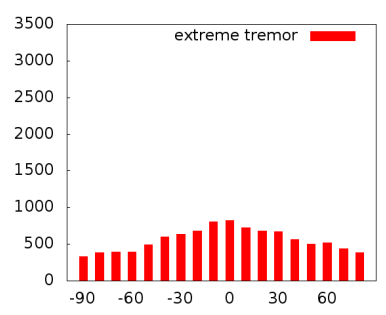

(d) extreme

Figure 2: Histograms of the relative line orientation (in bins of $10^{\circ}$ width) in tremor spiral images for different levels of tremor strength. The data is normalized with respect to the number of foreground pixels in each drawing.

\section{Evaluation data set}

For the evaluation 109 drawings from different patients with varying levels of tremor were available. They were manually divided in four groups (slight, medium, strong and extreme tremor). Information about this reference classification can be found in Tab. 1. The described method was performed for every group and the results were compared statistically.

\section{Results}

For all four tremor categories the histograms look very much alike within a class and show easily recognizable differences between classes. Representing examples of all categories are demonstrated in Fig. 2. Statistical values for a more detailed analysis are given in Tab. 1. All angle distributions are centered at approximately $0^{\circ}$, meaning the gradient points almost always directly away from the image center (as it is the case for a circle). This is even true for all but the most extreme cases of tremor.

The standard deviation, on the other hand, varies strongly when comparing two classes. These differences are highly significant $(\mathrm{p}<0.001$, Welch-Test). Assuming normal distributions we applied the Bayes decision rule to create class boundaries minimizing the number of errors in categorizing the images. The numbers given in Tab. 2 are optimal for the considered training set and resulted in a total of 24 misclassifications.

Table 1: Statistical results for Tremor examination data. Classes correspond to slight (1), medium (2), strong (3) and extreme (4) cases. Mean and standard deviation of relative orientation are given in ${ }^{\circ}$.

\begin{tabular}{lrrrr}
\hline & \multicolumn{4}{c}{ Class } \\
& $\mathbf{1}$ & $\mathbf{2}$ & $\mathbf{3}$ & $\mathbf{4}$ \\
\hline number of examples & 50 & 35 & 16 & 8 \\
mean of distribution & $2 \pm 1$ & $2 \pm 1$ & $3 \pm 1$ & $1 \pm 4$ \\
standard deviation & $15 \pm 1$ & $19 \pm 2$ & $28 \pm 9$ & $46 \pm 7$ \\
\hline
\end{tabular}

\section{Discussion}

The mean of the relative orientation is almost unaffected by tremor as it's effects are known to be dependent of the
Table 2: Class boundaries on standard deviation (in ${ }^{\circ}$ ) of relative orientation and classification errors (false positive / false negatives) for different levels of tremor

\begin{tabular}{lcc}
\hline Class & Boundaries & Errors \\
\hline 1 (slight tremor) & $<16.9$ & $6 / 2$ \\
2 (medium tremor) & $16.9-22.6$ & $9 / 9$ \\
3 (strong tremor) & $22.6-36.9$ & $4 / 12$ \\
4 (extreme tremor) & $>36.9$ & $5 / 1$ \\
\hline
\end{tabular}

movement directions and thus only certain parts of the spiral drawing will be subject to it. While these effects are mostly canceled out by calculating the mean, they still are visible in the standard deviation, because deviations from the ideal drawing path, both towards the center as well as away from it, will add up.

A simple automated classification was similar to the manual ordering, but still had an error rate of $22 \%$. A more elaborated classification method might be able to improve this. In addition, a larger study to collect more training data should be very beneficial.

However, our method already provides a proof of concept suitable for a quick check up. Especially, when considering that no more than a standardized spiral drawing paper, a scanner and a usual PC of low computing power are required to gain an objective result in less than 2 minutes.

\section{Acknowledgement}

We would like to thank PD Dr. P. H. Kraus of the St.JosefHospital in Bochum for providing the spiral images.

\section{Bibliography}

[1] P. Bain and L. Findley, Assessing Tremor Severity: A Clinical Handbook. Smith-Gordon, London, 1993.

[2] P. Kraus and A. Hoffmann, "Spiralometry: Computerized assessment of tremor amplitude on the basis of spiral drawing," Movement Disorders, vol. 25, no. 13, pp. 2164-2170, 2010.

[3] J.-M. Dolnitzki and S. Winter, "Merkmale aus zweidimensionalen Orientierungshistogrammen zur Beurteilung von Tremorspiralen," Proceedings of BVM 2012, pp. 322-327, 2012. 